Bull. Austral. Math. Soc.

Vol. 49 (1994) [257-265]

\title{
UNICITY THEOREMS FOR MEROMORPHIC OR ENTIRE FUNCTIONS
}

\author{
HONG-XUN YI
}

\begin{abstract}
In this paper, we prove that there exist three finite sets $S_{j}(j=1,2,3)$ such that any two non-constant meromorphic functions $f$ and $g$ satisfying $E_{f}\left(S_{j}\right)=E_{g}\left(S_{j}\right)$ for $j=1,2,3$ must be identical. As a particular case of the above result, we obtain that there exist two finite sets $S_{j}(j=1,2)$ such that any two non-constant entire functions $f$ and $g$ satisfying $E_{j}\left(S_{j}\right)=E_{g}\left(S_{j}\right)$ for $j=1,2$ must be identical, which answers a question posed by Gross.
\end{abstract}

\section{INTRODUCTION}

By a meromorphic function we shall always mean a meromorphic function in the complex plane. We use the usual notation of Nevanlinna theory of meromorphic functions as explained in [5]. We use $E$ to denote any set of positive real numbers of finite linear measure, not necessarily the same at each occurrence. We denote by $S(r, f)$ any quantity satisfying $S(r, f)=o(T(r, f))(r \rightarrow \infty, \quad r \notin E)$.

For any set $S$ and any meromorphic function $f$ let

$$
E_{f}(S)=\bigcup_{a \in S}\{z \mid f(z)-a=0\},
$$

where each zero of $f-a$ with multiplicity $m$ is repeated $m$ times in $E_{f}(S)$ (see [1]).

Nevanlinna proved the following well-known theorem.

Theorem A. (See $[6,4]$.) Let $S_{j}=\left\{a_{j}\right\}(j=1,2,3,4)$, where $a_{1}, a_{2}, a_{3}$ and $a_{4}$ are four distinct complex numbers $\left(a_{j}=\infty\right.$ is allowed). Suppose that $f$ and $g$ are non-constant meromorphic functions satisfying $E_{f}\left(S_{j}\right)=E_{g}\left(S_{j}\right)$ for $j=1,2,3,4$. Then either $f=g$, or $f$ is a linear fractional transformation of $g$, two of the values, say $a_{1}$ and $a_{2}$, must be Picard values, and the cross ratio $\left(a_{1}, a_{2}, a_{3}, a_{4}\right)=-1$.

Using Theorem $A$, the present author proved the following results.

Received 15 March 1993

Project supported by the National Natural Science Fundation of PRC.

Copyright Clearance Centre, Inc. Serial-fee code: 0004-9729/84 \$A2.00+0.00. 
TheOREM B. (See [7].) If, in addition to the assumptions of Theorem A,

$$
\frac{\left(2 a_{3}-a_{1}-a_{2}\right) a_{4}+\left(2 a_{1} a_{2}-a_{1} a_{3}-a_{2} a_{3}\right)}{\left(a_{2}-a_{1}\right)\left(a_{4}-a_{3}\right)} \neq-3,0,3,
$$

then $f=g$.

Theorem C. (See [7].) Let $S_{j}=\left\{a_{j}\right\}(j=1,2,3)$, where $a_{1}, a_{2}$ and $a_{3}$ are three distinct finite complex numbers. Suppose that $f$ and $g$ are two non-constant entire functions satisfying $E_{j}\left(S_{j}\right)=E_{g}\left(S_{j}\right)$ for $j=1,2,3$. If

$$
\frac{2 a_{3}-a_{1}-a_{2}}{a_{2}-a_{1}} \neq-3,0,3 \text {, }
$$

then $f=g$.

In [3] Gross also proved that there exist three finite sets $S_{j}(j=1,2,3)$ such that any two non-constant entire functions $f$ and $g$ satisfying $E_{f}\left(S_{j}\right)=E_{g}\left(S_{j}\right)$ for $j=1,2,3$ must be identical, and asked the following question (see [3, Question 6]): Can one find two finite sets $S_{j}(j=1,2)$ such that any two non-constant entire functions $f$ and $g$ satisfying $E_{f}\left(S_{j}\right)=E_{g}\left(S_{j}\right)$ for $j=1,2$ must be identical? Now it is natural to ask the following question: Can one find three finite sets $S_{j}(j=1,2,3)$ such that any two non-constant meromorphic functions $f$ and $g$ satisfying $E_{f}\left(S_{j}\right)=E_{g}\left(S_{j}\right)$ for $j=1,2,3$ must be identical?

Throughout this paper we shall use $w$ to denote the constant $\exp ((2 \pi i) /(n))$, where $n$ is a positive integer and $n>6$.

In this paper we answer the above questions. In fact, we prove more generally the following theorems.

THEOREM 1. Let $S_{1}=\left\{1, w, w^{2}, \ldots, w^{n-1}\right\}, S_{2}=\{a, b\}$ and $S_{3}=\{0\}$, where $a$ and $b$ are constants such that $a b \neq 0, a^{n} \neq b^{n}, a^{2 n} \neq 1, b^{2 n} \neq 1$ and $a^{n} b^{n} \neq 1$. Suppose that $f$ and $g$ are non-constant meromorphic functions satisfying $E_{f}\left(S_{j}\right)=$ $E_{g}\left(S_{j}\right)$ for $j=1,2,3$. Then $f=g$.

THEOREM 2. Let $S_{1}$ and $S_{2}$ be defined as in Theorem 1, and let $S_{3}=\{\infty\}$. Suppose that $f$ and $g$ are non-constant meromorphic functions satisfying $E_{f}\left(S_{j}\right)=$ $E_{g}\left(S_{j}\right)$ for $j=1,2,3$. Then $f=g$.

From Theorem 2 we immediately obtain the following result, which answers the question posed by Goss.

THeorem 3. Let $S_{1}$ and $S_{2}$ be defined as in Theorem 1. Suppose that $f$ and $g$ are non-constant entire functions satisfying $E_{f}\left(S_{j}\right)=E_{g}\left(S_{j}\right)$ for $j=1,2$. Then $f=g$.

The following interesting result will be needed in the proof of our theorems. 
Theorem 4. Let $S_{1}$ and $S_{3}$ be defined as in Theorem 2. Suppose that $f$ and $g$ are non-constant meromorphic functions satisfying $E_{f}\left(S_{j}\right)=E_{g}\left(S_{j}\right)$ for $j=1,3$. Then either $f=c g$, where $c^{n}=1$, or $f g=d$, where $d^{n}=1$.

\section{SOME LEMMAS}

Lemma 1. (See [8].) Let $f$ and $g$ be two non-constant meromorphic functions, and let $c_{1}, c_{2}$ and $c_{3}$ be three non-zero constants. If $c_{1} f+c_{2} g=c_{3}$, then

$$
T(r, f)<\bar{N}\left(r, \frac{1}{f}\right)+\bar{N}\left(r, \frac{1}{g}\right)+\bar{N}(r, f)+S(r, f) .
$$

Lemma 2. (See $[6,2]$.) Let $f_{1}, f_{2}, \ldots, f_{m}$ be linearly independent meromorphic functions satisfying $\sum_{j=1}^{m} f_{j}=1$. Then for $k=1,2, \ldots, m$ we have

$$
\begin{gathered}
T\left(r, f_{k}\right)<\sum_{j=1}^{m} N\left(r, \frac{1}{f_{j}}\right)+N\left(r, f_{k}\right)+N(r, D)-\sum_{j=1}^{m} N\left(r, f_{j}\right) \\
-N\left(r, \frac{1}{D}\right)+o(T(r)) \quad(r \notin E),
\end{gathered}
$$

where $D$ denotes the Wronskain

$$
D=\left|\begin{array}{cccc}
f_{1} & f_{2} & \cdots & f_{m} \\
f_{1}^{\prime} & f_{2}^{\prime} & \cdots & f_{m}^{\prime} \\
\ldots \ldots \ldots \ldots \ldots \ldots \ldots \ldots & \ldots \ldots \ldots \ldots \ldots \\
f_{1}^{(m-1)} & f_{2}^{(m-1)} & \cdots & f_{m}^{(m-1)}
\end{array}\right|
$$

and $T(r)$ denotes the maximum of $T\left(r, f_{j}\right), j=1,2, \ldots, m$.

LEMMA 3. (See [9].) Let $f_{1}, f_{2}$ and $f_{3}$ be three meromorphic functions satisfying $\sum_{j=1}^{3} f_{j}=1$, and let $g_{1}=-f_{3} / f_{2}, g_{2}=1 / f_{2}$ and $g_{3}=-f_{1} / f_{2}$. If $f_{1}, f_{2}$ and $f_{3}$ are linearly independent, then $g_{1}, g_{2}$ and $g_{3}$ are linearly independent.

\section{Proof of Theorem 4}

By the assumption, from Nevanlinna's second fundamental theorem, we have

$$
\begin{aligned}
(n-1) T(r, g) & <\sum_{k=0}^{n-1} N\left(r, \frac{1}{g-w^{k}}\right)+N(r, g)+S(r, g) \\
& =\sum_{k=0}^{n-1} N\left(r, \frac{1}{f-w^{k}}\right)+N(r, f)+S(r, g) \\
& <(n+1) T(r, f)+S(r, g) .
\end{aligned}
$$


Thus

$$
T(r, g)=O(T(r, f)) \quad(r \notin E) .
$$

Again by the assumption, we obtain

$$
f^{n}-1=e^{h}\left(g^{n}-1\right)
$$

where $h$ is an entire function. From (1) and (3), we have

$$
\begin{aligned}
T\left(r, e^{h}\right) & =T\left(r, \frac{f^{n}-1}{g^{n}-1}\right) \\
& <T\left(r, f^{n}\right)+T\left(r, g^{n}\right)+O(1) \\
& =n T(r, f)+n T(r, g)+O(1) \\
& <\left(n+\frac{n(n+1)}{n-1}\right) T(r, f)+S(r, f) .
\end{aligned}
$$

Thus

$$
T\left(r, e^{h}\right)=O(T(r, f)) \quad(r \notin E) .
$$

Let us put $f_{1}=f^{n}, f_{2}=e^{h}, f_{3}=-e^{h} g^{n}$, and let $T(r)$ denote the maximum of $T\left(r, f_{j}\right), j=1,2,3$. From (2), (3) and (4), we obtain

$$
\sum_{j=1}^{3} f_{j}=1
$$

and

$$
T(r)=O(T(r, f)) \quad(r \notin E) .
$$

Suppose that $f_{1}, f_{2}$ and $f_{3}$ are linearly independent. Applying Lemma 2 to the functions $f_{j}(j=1,2,3)$, from (5) and (6) we have

$$
\begin{gathered}
T\left(r, f_{1}\right)<\sum_{j=1}^{3} N\left(r, \frac{1}{f_{j}}\right)-N\left(r, \frac{1}{D}\right)+N(r, D)-N\left(r, f_{2}\right)-N\left(r, f_{3}\right) \\
+o(T(r, f)) \quad(r \notin E),
\end{gathered}
$$

where

$$
D=\left|\begin{array}{lll}
f_{1} & f_{2} & f_{3} \\
f_{1}^{\prime} & f_{2}^{\prime} & f_{3}^{\prime} \\
f_{1}^{\prime \prime} & f_{2}^{\prime \prime} & f_{3}^{\prime \prime}
\end{array}\right|
$$


We note that

$$
\sum_{j=1}^{3} N\left(r, \frac{1}{f_{j}}\right)=n N\left(r, \frac{1}{f}\right)+n N\left(r, \frac{1}{g}\right)
$$

and

$$
N\left(r, \frac{1}{D}\right) \geqslant n N\left(r, \frac{1}{f}\right)-2 \bar{N}\left(r, \frac{1}{f}\right)+n N\left(r, \frac{1}{g}\right)-2 \bar{N}\left(r, \frac{1}{g}\right) .
$$

From (5) and (8) we get

$$
D=\left|\begin{array}{ll}
f_{2}^{\prime} & f_{3}^{\prime} \\
f_{2}^{\prime \prime} & f_{3}^{\prime \prime}
\end{array}\right|
$$

and hence

$$
\begin{aligned}
N(r, D)-N\left(r, f_{2}\right)-N\left(r, f_{3}\right) & \leqslant N\left(r,\left(g^{n}\right)^{\prime \prime}\right)-N\left(r, g^{n}\right) \\
& =2 \bar{N}(r, g)=2 \bar{N}(r, f) .
\end{aligned}
$$

From (7), (9), (10) and (11) we deduce

$$
\begin{aligned}
n T(r, f) & <2 \bar{N}\left(r, \frac{1}{f}\right)+2 \bar{N}\left(r, \frac{1}{g}\right)+2 \bar{N}(r, g)+o(T(r, f)) \\
& <2 T(r, f)+4 T(r, g)+o(T(r, f)) \quad(r \notin E) .
\end{aligned}
$$

Let $g_{1}=-f_{3} / f_{2}=g^{n}, g_{2}=1 / f_{2}=e^{-h}$ and $g_{3}=-f_{1} / f_{2}=-e^{-h} f^{n}$. From (5) we obtain

$$
\sum_{j=1}^{3} g_{j}=1
$$

By Lemma 3 we know that $g_{1}, g_{2}$ and $g_{3}$ are linearly independent. In the same manner as above, we have

$$
n T(r, g)<4 T(r, f)+2 T(r, g)+o(T(r, f)) \quad(r \notin E) .
$$

Combining (12) and (13) we get

$$
(n-6) T(r, f)+(n-6) T(r, g)<o(T(r, f)) \quad(r \notin E) .
$$

Since $n>6,(14)$ is absurd. Hence $f_{1}, f_{2}$ and $f_{3}$ are linearly dependent. Then, there exist three constants $\left(c_{1}, c_{2}, c_{3}\right) \neq(0,0,0)$ such that

$$
c_{1} f_{1}+c_{2} f_{2}+c_{3} f_{3}=0
$$


If $c_{1}=0$, from (15) we have $c_{2} \neq 0, c_{3} \neq 0$ and

$$
f_{3}=-\frac{c_{2}}{c_{3}} f_{2}
$$

and hence

$$
g^{n}=\frac{c_{2}}{c_{3}}
$$

which is impossible. Thus $c_{1} \neq 0$ and

$$
f_{1}=-\frac{c_{2}}{c_{1}} f_{2}-\frac{c_{3}}{c_{1}} f_{3} .
$$

Now combining (5) and (16) we get

$$
\left(1-\frac{c_{2}}{c_{1}}\right) f_{2}+\left(1-\frac{c_{3}}{c_{1}}\right) f_{3}=1 .
$$

We discuss the following three cases.

(a) Assume $c_{1} \neq c_{2}$ and $c_{1} \neq c_{3}$. From (17) we have

$$
\left(1-\frac{c_{3}}{c_{1}}\right) g^{n}+e^{-h}=1-\frac{c_{2}}{c_{1}} \text {. }
$$

By Lemma 1 and (18) we obtain

$$
\begin{aligned}
n T(r, g) & <\bar{N}\left(r, \frac{1}{g}\right)+S(r, g) \\
& <T(r, g)+S(r, g),
\end{aligned}
$$

which is impossible.

(b) Assume $c_{1}=c_{3}$. From (17) we have $c_{1} \neq c_{2}$ and

$$
f_{2}=\frac{c_{1}}{c_{1}-c_{2}}
$$

that is,

$$
e^{h}=\frac{c_{1}}{c_{1}-c_{2}}
$$

From (5) and (19) we get

$$
f^{n}-\frac{c_{1}}{c_{1}-c_{2}} g^{n}=-\frac{c_{2}}{c_{1}-c_{2}}
$$


If $c_{2} \neq 0$, by Lemma 1 we have

$$
\begin{aligned}
n T(r, f) & <\bar{N}\left(r, \frac{1}{f}\right)+\bar{N}\left(r, \frac{1}{g}\right)+\bar{N}(r, f)+S(r, f) \\
& <2 T(r, f)+T(r, g)+S(r, f)
\end{aligned}
$$

and

$$
\begin{aligned}
n T(r, g) & <\bar{N}\left(r, \frac{1}{f}\right)+\bar{N}\left(r, \frac{1}{g}\right)+\bar{N}(r, g)+S(r, g) \\
& <T(r, f)+2 T(r, g)+S(r, g) .
\end{aligned}
$$

Hence,

$$
(n-3) T(r, f)+(n-3) T(r, g)<S(r, f)+S(r, g),
$$

which is impossible. Thus $c_{2}=0$. From (20) we deduce $f^{n}=g^{n}$ and $f=c g$, where $c^{n}=1$.

(c) Assume $c_{1}=c_{2}$. From (17) we have $c_{1} \neq c_{3}$ and

$$
f_{3}=\frac{c_{1}}{c_{1}-c_{3}}
$$

From (5) and (21) we get

$$
f_{1}+f_{2}=-\frac{c_{3}}{c_{1}-c_{3}}
$$

that is

$$
f^{n}+e^{h}=-\frac{c_{3}}{c_{1}-c_{3}}
$$

If $c_{3} \neq 0$, applying Lemma 1 to (22), we have

$$
\begin{aligned}
n T(r, f) & <\bar{N}\left(r, \frac{1}{f}\right)+S(r, f) \\
& <T(r, f)+S(r, f)
\end{aligned}
$$

which is impossible. Thus $c_{3}=0$. By (21) and (22) we deduce $f^{n}=-e^{h}, g^{n}=-e^{-h}$ and $f^{n} g^{n}=1$. Thus $f g=d$, where $d^{n}=1$.

This completes the proof of Theorem 4. 


\section{Proof of Theorem 2}

By the assumption $E_{f}\left(S_{j}\right)=E_{g}\left(S_{j}\right)(j=1,3)$, we have from Theorem 4 that $f=c g$, where $c^{n}=1$, or $f g=d$, where $d^{n}=1$. We discuss the following two cases.

(a) Suppose that

$$
f=c g \text {, }
$$

where $c^{n}=1$.

We discuss the following three subcases.

$\left(a_{1}\right)$ Assume that $a$ is not a Picard value of $f$, then there exists $z_{0}$ such that $f\left(z_{0}\right)=a$. By $E_{f}\left(S_{2}\right)=E_{g}\left(S_{2}\right)$, we obtain $g\left(z_{0}\right)=a$ or $g\left(z_{0}\right)=b$.

If $g\left(z_{0}\right)=a$, by (23) we have $a=c a$. Thus $c=1$, and $f=g$.

If $g\left(z_{0}\right)=b$, by (23) we have $a=c b$. Thus $a^{n}=c^{n} b^{n}=b^{n}$, which contradicts the assumption.

$\left(a_{2}\right)$ Assume that $b$ is not a Picard value of $f$. In the same manner as above, we have $f=g$.

(a $a_{3}$ Assume that $a$ and $b$ are Picard values of $f$. By $E_{f}\left(S_{2}\right)=E_{g}\left(S_{2}\right)$, we know that $a$ and $b$ are Picard values of $g$. Again by (23), we know that $c a$ and $c b$ are Picard values of $f$. Since a meromorphic function has at most two Picard values, then $a=c a$ or $a=c b$.

If $a=c a$, then $c=1$, and $f=g$. If $a=c b$, then $a^{n}=c^{n} b^{n}=b^{n}$, which contradicts the assumption.

(b) Suppose that

$$
f g=d,
$$

where $d^{n}=1$.

By the proof of Theorem 4, it is easy to see that 0 and $\infty$ are Picard values of $f$. Since a meromorphic function has at most two Picard values, then $a$ and $b$ are not Picard values of $f$. Thus there exists $z_{0}$ such that $f\left(z_{0}\right)=a$. By $E_{f}\left(S_{2}\right)=E_{g}\left(S_{2}\right)$, we obtain $g\left(z_{0}\right)=a$ or $g\left(z_{0}\right)=b$.

If $g\left(z_{0}\right)=a$, by (24) we have $a^{2}=d$. Thus $a^{2 n}=d^{n}=1$, which contradicts the assumption.

If $g\left(z_{0}\right)=b$, by (24) we have $a b=d$. Thus $a^{n} b^{n}=d^{n}=1$, which is also a contradiction.

This completes the proof of Theorem 2.

\section{Proof of Theorem 1}

Let $S_{4}=\{c, d\}$ and $S_{5}=\{\infty\}$, where $c=1 / a$ and $d=1 / b$. By the assumption, it is easy to see that $c d \neq 0, c^{n} \neq d^{n}, c^{2 n} \neq 1, d^{2 n} \neq 1$ and $c^{n} d^{n} \neq 1$. 
Let $F=1 / f$ and $G=1 / g . \quad B y E_{f}\left(S_{j}\right)=E_{g}\left(S_{j}\right)(j=1,2,3)$, we obtain $E_{F}\left(S_{j}\right)=E_{G}\left(S_{j}\right)(j=1,4,5)$. Applying Theorem 2 to the meromorphic functions $F$ and $G$, we have $F=G$. Thus $f=g$, which proves Theorem 1 .

\section{References}

[1] F. Gross, 'On the distribution of values of meromorphic functions', Trans. Amer. Math. Soc. 131 (1968), 199-214.

[2] F. Gross, Factorization of meromorphic functions (U.S. Govt. Printing Office Publication, Washington, D.C., 1972).

[3] F. Gross, 'Factorization of meromorphic functions and some open problems', in Complex analysis, Lecture Notes in Math. Vol. 599, (Proc. Conf. Univ. Kentucky, Lexington, Kentucky 1976) (Springer-Verlag, Berlin, Heidelberg, New York, 1977).

[4] G.G. Gundersen, 'Meromorphic functions that share four values', Trans. Amer. Math. Soc. 277 (1983), 545-567.

[5] W.K. Hayman, Meromorphic functions (Clarendon Press, Oxford, 1964).

[6] R. Nevanlinna, Le théorème de Picard-Borel et la théorie des fonctions méromorphes (Gauthier-Villars, Paris, 1929).

[7] Hong-Xun Yi, On a result of Gross (Shandong University Press, Jinan, 1990).

[8] Hong-Xun Yi, 'Uniqueness of meromorphic functions and a question of C.C. Yang', Complex variable 14 (1990), 169-176.

[9] Hong-Xun Yi, 'Meromorphic functions that share two or three values', Kodai Math. J. 13 (1990), 363-372.

Department of Mathematics

Shandong University

Jinan

Shandong 250100

People's Republic of China 